Article

\title{
Risk Aversion, Loss Aversion, and the Demand for Insurance
}

\author{
Louis Eeckhoudt ${ }^{1}$, Anna Maria Fiori ${ }^{2, *}$ (D) and Emanuela Rosazza Gianin 2 (D) \\ 1 Department of Economics and Quantitative Methods, IÉSEG School of Management, 3 rue de la Digue, \\ 59000 Lille, France; louis.eeckhoudt@fucam.ac.be \\ 2 Department of Statistics and Quantitative Methods, University of Milano-Bicocca, Via Bicocca degli \\ Arcimboldi 8, 20126 Milano, Italy; emanuela.rosazza1@unimib.it \\ * Correspondence: anna.fiori@unimib.it; Tel.: +39-02-6448-3112
}

Received: 27 March 2018; Accepted: 22 May 2018; Published: 25 May 2018

\begin{abstract}
In this paper we analyze insurance demand when the utility function depends both upon final wealth and the level of losses or gains relative to a reference point. Besides some comparative statics results, we discuss the links with first-order risk aversion, with the Omega measure, and with a tendency to over-insure modest risks that has been been extensively documented in real insurance markets.
\end{abstract}

Keywords: first-order risk aversion; stochastic dominance; insurance; expected utility

\section{Introduction}

In his early and deep contributions to the insurance economics literature, Borch $(1960,1962)$ made the assumption that the decision-maker's utility function depends only upon his/her final wealth. This assumption was adopted for decades in the analysis of insurance choice, and a recent survey of this literature by Schlesinger (2013) confirms this observation.

Borch's assumption has been considered and developed in a number of papers, also in conjunction with Yaari (1987) dual theory of choice and its implications for insurance demand (see, for instance, Denneberg 1990; Doherty and Eeckhoudt 1995; Wang and Young 1998).

An exception to Borch's assumptions resulted from the distinction made by Segal and Spivak (1990) between second-order and first-order risk aversion (respectively, SORA and FORA). While under SORA (as for Borch) the utility function of final wealth is always differentiable, it exhibits (at least) one point of non-differentiability under FORA, and this assumption has important consequences for the insured's optimal behavior. For instance, under FORA, Mossin's (1968) famous claim about the non-optimality of full insurance under a positive premium loading may no longer hold.

More recently, Guo et al. (2016) have proposed a model of choice in which the decision-maker's risk-averse utility function depends upon two arguments: his/her final wealth and the level of losses or gains relative to a reference level. ${ }^{1}$ Guo et al. (2016) consider only the implications of their model for the stochastic dominance literature and do not discuss the implications for insurance demand.

It is precisely the purpose of the present paper to analyze the implications of Guo et al.'s (2016) model for the analysis of insurance decisions. It is worth observing that Guo's model provides new

1 As pointed out by a referee, Guo's model contains indeed two deviations from Borch's assumptions: one is the presence of gain/loss utility, the other one is non-differentiability at the reference point. In an earlier study, Eeckhoudt et al. (2016) have considered a gain/loss argument of utility where differentiability holds everywhere. Other authors, contrarily to Guo et al. (2016), have assumed that at least one component of the utility function exhibits risk loving (see, e.g., Bernard and Ghossoub 2010; Köbberling and Wakker 2005, and the literature therein). 
perspectives about optimal behavior with respect to insurance choices. Additionally, since Guo's model presents some similarities (but also differences) with the FORA case developed by Segal and Spivak (1990), we also extend a result derived by Segal and Spivak (1990) for binary risks to any distribution of losses.

In the context of proportional insurance, this extension creates a link between features of the insurance contract and the Omega performance measure, a concept often used in the quantitative finance literature (see, e.g., Keating and Shadwick 2002). Based on the Omega measure, we obtain a necessary and sufficient condition for the optimality of full coverage and illustrate its similarities with some recent works on stochastic orderings (Müller et al. 2016) and optimal portfolio choices (Bernard and Ghossoub 2010).

A necessary and sufficient condition for the optimality of full coverage is also derived in the context of deductible insurance, in which the extension of Segal and Spivak's result to a general loss distribution is shown to be possible under Guo's model.

Based on a comparative analysis of insurance decisions under FORA and SORA, we prove that FORA always stimulates the demand for insurance coverage relative to SORA, leading to either a higher co-insurance rate or a lower level of deductible. These findings can help explain some empirical "anomalies" in risky choices that have been documented for some agents in real insurance markets, namely that people tend to over-insure against modest risks (Schmidt 2016) and that many customers purchase low deductibles despite costs exceeding expected losses (Sydnor 2010).

Our analysis differs from Schmidt (2016) substantially. Whereas Schmidt (2016) uses a single-argument value function specified as a power function of gains and losses, our results are derived for a two-argument utility model and for a general specification of the gain/loss function (not necessarily power-based). Different than Schmidt (2016), we work with general loss distributions (rather than with binary risks) and we allow a positively loaded insurance premium. Our approach is therefore instrumental to analyze the impact of FORA on the decision to take up full coverage when insurance contracts are priced above their actuarially fair value, as usually happens in real-world circumstances.

In Guo's model, preferences are consistent with a set of stochastic dominance rules for ordering risks in the presence of an exogenous target. Based on these rules, we prove an extension of Arrow's theorem of the deductible (Arrow 1974) showing that, under FORA, risk-averse decision-makers maintain a preference for concentrating insurance coverage in the states with the largest losses. Hence, a straight deductible policy is proved to be the optimal choice relative to other forms of insurance contracts.

Guo et al. (2016) were not the first to propose the reference-dependent utility model. Its origins date back to Köszegi and Rabin $(2006,2007)$, whose work stimulated an impressive stream of theoretical research aimed at investigating how FORA is embedded in alternative models of non-expected utility (see Masatlioglu and Raymond (2016), for a complete taxonomy). Our framework is, on the one hand, more specific than that of Masatlioglu and Raymond and permits the derivation of some relevant results under milder conditions. On the other hand, our setting is a bit different from that of the aforementioned authors since we consider an exogenous reference point instead of an endogenous one.

Other important precursors of Guo et al. (2016) are the papers of Cox and Sadiraj (2006, 2008), Schmidt et al. (2008), and Sugden (2003), who all pointed out an "income-and-wealth" characterization of utility. In particular, a promising direction for future research is the "Third-generation prospect theory" introduced by Schmidt et al. (2008) combining reference dependence, decision weights, and an uncertain reference point. Based on these considerations, we believe that it may be useful to explore the implications of allowing a two-argument utility function for insurance demand, and we show here that the model of Guo et al. (2016) provides a tractable framework for this analysis.

Our paper is organized as follows. In Section 2, we describe the model and its specificities for the measurement of local risk aversion. Proportional insurance is covered in Section 3, while in 
Section 4 we turn to deductible insurance. Alternative forms of insurance contracts are compared in Section 5. Section 6 includes a discussion of our findings in connection with some recent literature and a few conclusions.

\section{Model and Notation}

A decision-maker $(\mathrm{DM})$ with initial wealth $w_{0}$ is exposed to a random loss $L$ defined on $[0, \bar{l}]$ where $\bar{l} \leq w_{0}$ is the potential maximum loss. We denote by $F_{L}(l)$ the cumulative distribution function of $L$ and by $\mu_{L}$ its expected value. Initial wealth is assumed fixed and known to the DM. ${ }^{2}$

The DM may purchase an insurance contract that compensates the occurrence of a loss by an indemnity $I(L)$, with $0 \leq I(L) \leq \bar{l}$. The premium charged for the contract is assumed proportional to the expected indemnity, $\pi=(1+k) E[I(L)]$, where $k>0$ is the loading factor. The final wealth of an insured agent is thus given by $W=w_{0}-L+I(L)-\pi$.

Under FORA, the DM's utility function is concave with (at least) one point of non-differentiability. Such a situation may arise because of utility loss aversion ${ }^{3}$ as recently argued by Guo et al. (2016). Their model combines a classical outcome-based utility function, which reflects intrinsic taste for end-period wealth, with a Kahneman-Tversky value function, which describes the sensation of gain or loss induced by deviations from a reference wealth level. Formally, the DM's reference-dependent utility is specified by

$$
v(w)=u(w)+\Re^{\eta, \lambda}[u(w)-u(r)]
$$

where $r$ is a fixed (exogenous) reference point, $u$ is a concave utility function of wealth, and $\Re^{\eta, \lambda}$ is a piecewise linear value function:

$$
\Re^{\eta, \lambda}(x)= \begin{cases}\eta x & \text { for } x \geq 0 \\ \lambda \eta x & \text { for } x<0\end{cases}
$$

Here, $\eta$ is a positive parameter that represents the relative weight of gain-loss utility, while $\lambda>1$ is the magnitude of loss aversion. In the limit case of $\eta \rightarrow \infty$, the reference-dependent utility expressed by Equation (1) is only composed of a Kahneman-Tversky value function (see Guo et al. (2016), for a formal proof). For computational purposes, the utility function expressed by Equation (1) may be rewritten as follows:

$$
v(w)= \begin{cases}v_{+}(w)=(1+\eta) u(w)-\eta u(r) & \text { for } w \geq r \\ v_{-}(w)=(1+\lambda \eta) u(w)-\lambda \eta u(r) & \text { for } w<r\end{cases}
$$

For reference-dependent utility models, the choice of the target or reference wealth $r$ plays a central role. In a paper dealing with portfolio choice under cumulative prospect theory, Bernard and Ghossoub (2010) argue that the target corresponds to "the amount the individual would have received at the end of the period had he invested all of his initial wealth in the risk-free asset." In a sense in the portfolio problem, the target corresponds to the choice of a zero risk situation. The equivalent target in an insurance problem is a situation, where the DM is fully insured, so one interesting way to define the reference wealth $r$ is given by

$$
r=w_{0}-(1+k) \mu_{L}
$$

2 This assumption is further discussed in the last section, in which we also outline some papers proposing possible generalizations.

3 As pointed out by a referee, the concept of utility loss aversion is different from the probabilistic loss aversion that comes in cumulative prospect theory from any differences in decision weights between gains and losses (see Schmidt and Zank 2008). In the present work, the term loss aversion is used in the sense of utility loss aversion. 
This reference point was also considered in an insurance setting by Schmidt (2016), who refers to Equation (4) as the "safe alternative".

Indeed, the definition of the reference point is critical and may be a subjective choice. Alternative approaches were suggested, for instance, by Köszegi and Rabin $(2006,2007)$ (who considered as a reference point for practical purposes the expected value of a lottery), by Gul (1991) (who used the certainty equivalent), and by Schmidt et al. (2008) (who argued in favor of an uncertain reference point). A variety of possible characterizations for the reference point were empirically tested by Baillon et al. (2016), who found that approximately $60 \%$ of subjects involved in an experimental study chose a fixed reference point consisting of either the status quo (given by the participation fee paid to subjects enrolled in the study), or a security level (given by the maximum of the minimal outcome of the prospects in a choice). As is common practice in empirical studies, the experiment of Baillon et al. (2016) involved choices among speculative gambles, i.e., lotteries in which the risk could result in either a loss or a gain to the agent.

As pointed out by Sydnor (2010), insurance decisions are different from speculative gambles because their consequences are determined entirely in the loss domain. It is therefore questionable whether the status quo (corresponding to initial wealth without taking up insurance) could be an appropriate reference point for a two-argument utility model. Conversely, a safe alternative is available if subjects take up full insurance (Schmidt 2016), and some empirical evidence reported in Baillon et al. (2016) is also consistent with a security-based rule according to which subjects adopt the maximum outcome they can reach for sure as the reference point (see Baillon et al. (2016), footnote 4 and online appendix).

In the following sections, we analyze optimal insurance demand using Equation (4) as the reference point. A comparative statics analysis is included in Section 3 to illustrate the impact of changes in $r$.

\section{First-Order Risk Aversion, Loss Aversion, and Local Risk Attitude}

The reference-dependent utility $v$ specified in Equation (1) is always concave ${ }^{4}$ owing to the concavity of $u$ and the piecewise linearity of the value function $\Re^{\eta, \lambda}$. However, in contrast with recent literature on downside loss aversion within the EU framework (see, e.g., Eeckhoudt et al. (2016); Jarrow and Zhao (2006); references therein), neither loss aversion nor the gain-loss component introduced via Equation (2) affect the degree of local risk aversion of $u$ away from the reference point. It is straightforward to verify that, for all $w \neq r$, the Arrow-Pratt coefficient of absolute risk aversion (ARA) for $v$ is given by

$$
A R A_{v}(w)=-\frac{v^{\prime \prime}(w)}{v^{\prime}(w)}=-\frac{u^{\prime \prime}(w)}{u^{\prime}(w)}=A R A_{u}(w) .
$$

We now examine local risk attitudes at the reference point considering a real number $t$ and a zero-mean risk $\varepsilon$ with cumulative distribution function $G_{\varepsilon}$ valued in $(-\infty,+\infty)$. Denote by $\pi_{v}(r, t)$ the risk premium that a DM with utility function expressed by Equation (3) associates to the outcome $r+t \varepsilon$. This risk premium is defined by

$$
v\left[r-\pi_{v}(r, t)\right]=E[v(r+t \varepsilon)]=(1-p) E[v(r+t \varepsilon) \mid \varepsilon \leq 0]+p E[v(r+t \varepsilon) \mid \varepsilon>0]
$$

where $p=P(\varepsilon>0)=1-G_{\varepsilon}(0)$.

4 Notice the difference with non-EU models of loss aversion where the utility function has convex portions. 
In accordance with Davies and Satchell (2007), we examine the characteristics of $\pi_{v}$ for sufficiently small risks by taking a first-order Taylor approximation around the reference point on the left-hand side (LHS) of the above equation:

$$
L H S \approx v(r)-\pi_{v}(r, t) v_{-}^{\prime}(r) \approx u(r)-\pi_{v}(r, t)(1+\lambda \eta) u^{\prime}(r)
$$

and a second-order approximation around $r$ on the right-hand side (RHS):

$$
R H S \approx(1-p) E\left[v_{-}(r)+t \varepsilon v_{-}^{\prime}(r)+\frac{t^{2} \varepsilon^{2}}{2} v_{-}^{\prime \prime}(r) \mid \varepsilon \leq 0\right]+p E\left[v_{+}(r)+t \varepsilon v_{+}^{\prime}(r)+\frac{t^{2} \varepsilon^{2}}{2} v_{+}^{\prime \prime}(r) \mid \varepsilon>0\right] .
$$

For notational simplicity we denote the conditional mean and variance of $\varepsilon$, given $\varepsilon \leq 0$, as $\mu_{\varepsilon}^{-}$ and $\sigma_{\varepsilon}^{2,-}$, and, given $\varepsilon>0$, as $\mu_{\varepsilon}^{+}$and $\sigma_{\varepsilon}^{2,+}$, respectively. Based on Equation (3), the approximation on the RHS above can be rewritten as

$$
\begin{aligned}
R H S \approx & (1-p)\left[v_{-}(r)+t(1+\lambda \eta) u^{\prime}(r) \mu_{\varepsilon}^{-}+\frac{t^{2}}{2}(1+\lambda \eta) u^{\prime \prime}(r) \sigma_{\varepsilon}^{2,-}\right] \\
& +p\left[v_{+}(r)+t(1+\eta) u^{\prime}(r) \mu_{\varepsilon}^{+}+\frac{t^{2}}{2}(1+\eta) u^{\prime \prime}(r) \sigma_{\varepsilon}^{2,+}\right] \\
\approx & u(r)-t u^{\prime}(r) \eta(\lambda-1) p \mu_{\varepsilon}^{+}+\frac{t^{2}}{2} u^{\prime \prime}(r)\left[(1+\lambda \eta)(1-p) \sigma_{\varepsilon}^{2,-}+(1+\eta) p \sigma_{\varepsilon}^{2,+}\right]
\end{aligned}
$$

where $(1-p) \mu_{\varepsilon}^{-}=-p \mu_{\varepsilon}^{+}$because $\varepsilon$ is a zero-mean risk, and $v_{-}(r)=v_{+}(r)=u(r)$.

From $L H S \approx R H S$, we finally obtain

$$
\pi_{v}(r, t)=\underbrace{t \frac{\eta(\lambda-1)}{1+\lambda \eta} p \mu_{\varepsilon}^{+}}_{\pi_{v, 1}(r, t)}+\underbrace{\frac{t^{2}}{2} A R A_{u}(r)\left[(1-p) \sigma_{\varepsilon}^{2,-}+\frac{1+\eta}{1+\lambda \eta} p \sigma_{\varepsilon}^{2,+}\right]}_{\pi_{v, 2}(r, t)} .
$$

Based on Equation (5), we may decompose the total risk premium $\pi_{v}(r, t)$ into the sum of two terms. The first term $\pi_{v, 1}(r, t)$ represents the first-order effect of loss aversion on risk attitude. The sign of this effect is always positive as long as $\eta>0$ and $\lambda>1$. Differentiating both sides of Equation (5) with respect to $t$, we obtain, at $t=0^{+}$,

$$
\left.\frac{\partial \pi_{v}(r, t)}{\partial t}\right|_{t=0^{+}}=\frac{\eta(\lambda-1)}{1+\lambda \eta} p \mu_{\varepsilon}^{+}
$$

which is a special case of Segal and Spivak's (1990) result ${ }^{5}$ at points where the utility function is not differentiable. This shows that, in the reference-dependent utility model of Guo et al. (2016), the risk premium $\pi_{v}(r, t)$ for small risks is proportional to $t$ rather than $t^{2}$, i.e., loss aversion induces FORA at $r$.

The second term $\pi_{v, 2}(r, t)$ shows the effect of curvature of the utility function $v$ in Guo's model in Equation (3) and is analogous to the classical Arrow-Pratt risk premium associated to the basic utility $u$ :

$$
\pi_{u}(r, t)=\frac{t^{2}}{2} A R A_{u}(r) \sigma_{\varepsilon}^{2}
$$

where $\sigma_{\varepsilon}^{2}=(1-p) \sigma_{\mathcal{\varepsilon}}^{2,-}+p \sigma_{\mathcal{\varepsilon}}^{2,+}$ is the total variance of $\varepsilon$. Comparing Equation (6) with $\pi_{v, 2}$, it appears that

$$
\pi_{v, 2}(r, t)=\pi_{u}(r, t)-\frac{t^{2}}{2} A R A_{u}(r) \frac{\eta(\lambda-1)}{1+\lambda \eta} p \sigma_{\varepsilon}^{2,+}
$$

5 see (Segal and Spivak (1990), p. 118). 
so the presence of loss aversion lowers the effect of utility curvature by a factor that depends on the upper variance $\sigma_{\mathcal{\varepsilon}}^{2,+}$ of the zero-mean risk. This reflects the change in (second-order) risk perception across the reference point induced by loss aversion.

Moreover, by Equations (5) and (6),

$$
\pi_{v}(r, t)-\pi_{u}(r, t)=p \frac{\eta(\lambda-1)}{1+\lambda \eta}\left[t \mu_{\varepsilon}^{+}-\frac{t^{2}}{2} A R A_{u}(r) \sigma_{\varepsilon}^{2,+}\right],
$$

and, consequently,

$$
\pi_{v}(r, t) \geq \pi_{u}(r, t) \Leftrightarrow 0<t<\frac{2 \mu_{\varepsilon}^{+}}{\sigma_{\varepsilon}^{2,+} A R A_{u}(r)} .
$$

Since the focus of our analysis is on small risks, i.e., around $t=0$, Equation (8) implies that loss aversion induces higher risk aversion at $r$.

As Segal and Spivak (1990) have pointed out, for small risks, a DM is almost risk-neutral under SORA (i.e., for $\lambda=1$ in Guo's model) because a differentiable utility function is almost linear in a neighborhood of $r$. As the Arrow-Pratt risk premium expressed by Equation (6) under SORA is proportional to $t^{2}$, the DM evaluates small risks essentially by their expected value and is always willing to take up very small gambles that are in his/her favor.

Conversely, a DM may reject a small gamble under FORA if the expected yield is positive but sufficiently small, which is a consequence of his/her risk attitude being of order 1. By similar arguments, Segal and Spivak (1990) have argued (and have proved for binary risks) that people may buy full insurance under FORA even though they have to pay some marginal loading. This behavior will be studied in detail in Sections 3 and 4, where we will extend Segal's and Spivak's result to a general loss distribution using Guo's model and distinguishing the case of proportional insurance from that of deductible insurance.

It is interesting to observe that the first-order term of the total risk premium expressed by Equation (5) is increasing in each of the two "behavioral" parameters $\lambda, \eta$. As long as $\eta>0$ and $\lambda>1$, differentiating $\pi_{v, 1}(r, t)$ with respect to $\lambda$ (respectively, $\eta$ ) gives

$$
\begin{aligned}
& \frac{\partial \pi_{v, 1}(r, t)}{\partial \lambda}=t \frac{\eta(1+\eta)}{(1+\lambda \eta)^{2}} p \mu_{\varepsilon}^{+}>0 \\
& \frac{\partial \pi_{v, 1}(r, t)}{\partial \eta}=t \frac{\lambda-1}{(1+\lambda \eta)^{2}} p \mu_{\varepsilon}^{+}>0 .
\end{aligned}
$$

Evaluating the overall effect of each parameter on Equation (5), we obtain

$$
\begin{aligned}
& \frac{\partial \pi_{v}(r, t)}{\partial \lambda}=\frac{\partial \pi_{v, 1}(r, t)}{\partial \lambda}+\frac{\partial \pi_{v, 2}(r, t)}{\partial \lambda}=t \frac{\eta(1+\eta)}{(1+\lambda \eta)^{2}} p\left[\mu_{\varepsilon}^{+}-\frac{t}{2} A R A_{u}(r) \sigma_{\varepsilon}^{2,+}\right] \\
& \frac{\partial \pi_{v}(r, t)}{\partial \eta}=\frac{\partial \pi_{v, 1}(r, t)}{\partial \eta}+\frac{\partial \pi_{v, 2}(r, t)}{\partial \eta}=t \frac{\lambda-1}{(1+\lambda \eta)^{2}} p\left[\mu_{\varepsilon}^{+}-\frac{t}{2} A R A_{u}(r) \sigma_{\varepsilon}^{2,+}\right]
\end{aligned}
$$

which are both positive under the condition expressed by Equation (8) discussed above. It thus appears that higher values of $\lambda, \eta$ are associated to a higher risk premium that a FORA agent is willing to pay to avoid a sufficiently small fair gamble when $w=r$. The intuition underlying these findings is that the first-order effect of an increase in $\lambda$ induces a drop in $v_{-}(w)$ that is not compensated by $v_{+}(w)$. The second-order effect of $\lambda$ is more complex; however, as long as we are sufficiently close to the reference point (i.e., under the condition expressed by Equation (8)), it is the first-order effect that prevails. A similar intuition can also be recovered for the impact of $\eta$. 


\section{Proportional Insurance}

We here suppose that insurance is available in the form of proportional coverage (or coinsurance). We characterize the optimal insurance-purchasing decision of a DM with a reference-dependent utility, expressed by (1), and a reference wealth $r$ corresponding to the no-risk situation outlined in Equation (4). The impact of a modification in preferences or in $r$ will be studied in Section 3.

A DM who insures a fraction $\alpha$ of his/her initial wealth ${ }^{6}$, with $0 \leq \alpha \leq 1$, is charged a proportional premium:

$$
\pi=(1+k) \alpha \mu_{L}
$$

so his / her final wealth is given by

$$
W(L, \alpha)=w_{0}-L+\alpha L-\pi(L)=w_{0}-(1-\alpha) L-(1+k) \alpha \mu_{L}
$$

Based on Equation (1), the DM chooses the optimal proportion of insurance coverage $\alpha_{r}^{*} \in[0,1]$ by maximizing the expected utility of the final wealth specified in Equation (10):

$$
\begin{aligned}
V(\alpha) & =E[v(W(L, \alpha))]=E\left\{u(W)+\Re^{\eta, \lambda}[u(W)-u(r)]\right\} \\
& =\int_{0}^{l^{r}}\{u(w(l, \alpha))+\eta[u(w(l, \alpha))-u(r)]\} d F_{L}(l) \\
& +\int_{l^{r}}^{\bar{l}}\{u(w(l, \alpha))+\lambda \eta[u(w(l, \alpha))-u(r)]\} d F_{L}(l)
\end{aligned}
$$

where $l^{r}=(1+k) \mu_{L}$ is the loss incurred at the reference wealth $r$ (henceforth, the target loss), such that $l \leq l^{r}$ iff $w \geq r$.

Notice that, under FORA, the utility function $v$ is not differentiable at $w=r$, nor therefore at $L=l^{r}$. Nevertheless, assuming that $L$ is continuous at $l^{r}$ (together with Lebesgue's dominated convergence theorem) guarantees that

$$
V^{\prime}(\alpha)=(1+\eta) \int_{0}^{l^{r}} u^{\prime}(w(l, \alpha)) w_{\alpha} d F_{L}(l)+(1+\lambda \eta) \int_{l^{r}}^{\bar{l}} u^{\prime}(w(l, \alpha)) w_{\alpha} d F_{L}(l)
$$

where $w_{\alpha}=\frac{\partial w}{\partial \alpha}=l-(1+k) \mu_{L}$. The previous equation can be also written as

$$
\begin{aligned}
V^{\prime}(\alpha) & =(1+\eta) \int_{0}^{\bar{l}} u^{\prime}(w(l, \alpha)) w_{\alpha} d F_{L}(l)+(\lambda-1) \eta \int_{l^{r}}^{\bar{l}} u^{\prime}(w(l, \alpha)) w_{\alpha} d F_{L}(l) \\
& =(1+\eta) U^{\prime}(\alpha)+(\lambda-1) \eta \int_{l^{r}}^{\bar{l}} u^{\prime}(w(l, \alpha)) w_{\alpha} d F_{L}(l)
\end{aligned}
$$

where

$$
U(\alpha)=E[u(W(L, \alpha))]=\int_{0}^{\bar{l}} u(w(l, \alpha)) d F_{L}(l)
$$

is the classical expected utility of terminal wealth and $U^{\prime}(\alpha)=\int_{0}^{\bar{l}} u^{\prime}(w(l, \alpha)) w_{\alpha} d F_{L}(l)$. It thus appears that $V^{\prime}(\alpha)$ can be decomposed into a first term based on EU maximization with respect to the initial utility function $u$ and a second term taking into account gain-loss utility à la Guo et al. (2016) (hence considering both $\lambda$ and $\eta$ ).

6 Since it is often the case that over insurance is not allowed, we maintain throughout the paper the assumption that the coinsurance rate $\alpha$ cannot exceed 1. As pointed out by a referee, this is also known as the principle of indemnity in the insurance economics literature (see Peter et al. 2017). 
Proposition 1. Consider an EU-maximizing agent whose reference-dependent utility, expressed by Equation (1), exhibits FORA at the target wealth $r$ specified in Equation (4). Define the lower and upper partial expectations of $L$ with respect to the target loss $l^{r}=(1+k) \mu_{L}$ as, respectively,

$$
\begin{aligned}
& E\left[\left(l^{r}-L\right)^{+}\right]=\int_{0}^{l^{r}}\left(l^{r}-l\right) d F_{L}(l), \\
& E\left[\left(L-l^{r}\right)^{+}\right]=\int_{l^{r}}^{\bar{l}}\left(l-l^{r}\right) d F_{L}(l),
\end{aligned}
$$

where $F_{L}$ is assumed continuous at $l^{r}$.

If

$$
\frac{1+\lambda \eta}{1+\eta}>\frac{E\left[\left(l^{r}-L\right)^{+}\right]}{E\left[\left(L-l^{r}\right)^{+}\right]}
$$

the agent will purchase full insurance coverage, i.e., $\alpha_{r}^{*}=1$, even when the insurance premium has a positive loading $k>0$.

If the opposite inequality holds in Equation (17), then the agent will purchase partial insurance coverage, i.e., $0 \leq \alpha_{r}^{*}<1$.

Finally, if equality holds in the condition expressed by Equation (17) and $V$ is a strictly concave function, then the agent will also purchase full insurance coverage, i.e., $\alpha_{r}^{*}=1$.

Proof. Based on Equation (11), we notice that the expected utility $V(\alpha)$ is a concave function of the decision variable $\alpha$. The optimal level of insurance coverage $\alpha_{r}^{*}$ is consequently found by studying the sign of $V^{\prime}(\alpha)$ in Equation (12). Since $w(l, 1)=w_{0}-(1+k) \mu_{L}=r$, evaluating $V^{\prime}(\alpha)$ at $\alpha=1$ gives

$$
\begin{aligned}
V^{\prime}(1) & =u^{\prime}(r)\left[(1+\eta) \int_{0}^{l^{r}}\left(l-l^{r}\right) d F_{L}(l)+(1+\lambda \eta) \int_{l^{t}}^{\bar{l}}\left(l-l^{r}\right) d F_{L}(l)\right] \\
& =u^{\prime}(r)\left\{-(1+\eta) E\left[\left(l^{r}-L\right)^{+}\right]+(1+\lambda \eta) E\left[\left(L-l^{r}\right)^{+}\right]\right\},
\end{aligned}
$$

which is strictly positive under the condition expressed by Equation (17). It follows that $\alpha_{r}^{*}=1$ is a constrained optimum (when over insurance is prohibited). The last two statements follow immediately from the sign of $V^{\prime}(1)$ and from the concavity of $V$.

Under the general definition of loss aversion as the behavioral phenomenon that losses matter more than gains, the ratio $\frac{1+\lambda \eta}{1+\eta}$, which determines the occurrence of the condition expressed by Equation (17), may be interpreted as an index of loss aversion in the style of Köbberling and Wakker (2005). It is the magnitude of this index that can induce a rational, EU-maximizing agent to purchase full insurance coverage at a loaded price in violation of Mossin's theorem but in accordance with common observation for some agents (see, e.g., Borch 1990). Our result shows that the amount of loss aversion required to trigger the realization of Equation (17) is determined by the partial moment ratio $\frac{E\left[\left(l^{r}-L\right)^{+}\right]}{E\left[\left(L-l^{r}\right)^{+}\right]}$, which compares the DM's upside potential (the expectation of loss deviations below the threshold $l^{r}$, in the numerator) to his/her downside risk exposure (the expectation of loss deviations in excess of $l^{r}$, in the denominator). This moment ratio is the analogue of a portfolio performance index that is well known in the quantitative finance literature as the Omega measure (see, e.g., Bertrand and Prigent (2011) and the references therein). We will accordingly denote the RHS of Equation (17) by

$$
\Omega\left(l^{r}\right)=\frac{E\left[\left(l^{r}-L\right)^{+}\right]}{E\left[\left(L-l^{r}\right)^{+}\right]} .
$$

In the context of investment decisions, a (subjectively modified) version of the Omega measure has been shown to determine the optimal portfolio choice of a cumulative prospect theory (CPT) agent in a one-period economy with one risk-free and one risky asset (Bernard and Ghossoub 2010). More recently, 
the Omega measure expressed by Equation (18) has appeared in a stochastic dominance rule of order $(1+\gamma)$ that Müller et al. (2016) have introduced to explain why some classes of utility-maximizing investors prefer to purchase a risk-free rather than a risky asset (or portfolio). As far as we know, our condition expressed by Equation (17) represents the first evidence of a link between the Omega measure and the optimal insurance choice.

Based on the decomposition,

$$
\underbrace{\mu_{L}-l^{r}}_{-k \mu_{L}}=\underbrace{\int_{0}^{l^{r}}\left(l-l^{r}\right) d F_{L}}_{-E\left[\left(l^{r}-L\right)^{+}\right]}+\underbrace{\int_{l^{r}}^{\bar{l}}\left(l-l^{r}\right) d F_{L}}_{E\left[\left(L-l^{r}\right)^{+}\right]},
$$

the Omega measure expressed by Equation (18) may be rewritten as

$$
\Omega\left(l^{r}\right)=1+k \frac{\mu_{L}}{E\left[\left(L-l^{r}\right)^{+}\right]},
$$

which implies that $\Omega\left(l^{r}\right)=1$ if and only if $k=0$. This leads to an optimality of full coverage when insurance is fairly priced, since the loss aversion measure $\frac{1+\lambda \eta}{1+\eta}$ - which appears on the LHS of Equation (17) — is greater than 1 as long as $\lambda>1$ and $\eta>0$. Additionally, from

$$
\frac{\partial E\left[\left(L-l^{r}\right)^{+}\right]}{\partial k}=-\mu_{L}\left[1-F_{L}\left(l^{r}\right)\right]<0,
$$

it follows that $\Omega\left(l^{r}\right)$ is an increasing function of $k$ and exceeds 1 as long as the insurance premium is positively loaded. Consequently, for given values of the behavioral parameters $\lambda>1$ and $\eta>0$, there is a range of values of the loading factor $k$ such that the condition expressed by Equation (17) is verified for $0 \leq k \leq k^{*}$, leading to the optimality of full insurance coverage. The value of $k^{*}$ can be determined from Equation (17) once the shape of the loss distribution $F_{L}$ is specified.

An empirical study of Guo's model is beyond the aim of the present paper. However, we refer the interested reader to papers that have estimated similar models, finding values of $\lambda$ either in the range of 1.4-5 (see Table 1 in Abdellaoui et al. (2007)) or slightly above 1 Murphy and ten Brincke (2018); Nilsson et al. (2011). For a careful and critical review, see, e.g., Harrison and Swarthout (2016).

\section{Comparative Statics Results}

An interesting question relates to the comparison of the optimal coverage proportions implied by FORA in the reference-dependent utility model of Guo et al. (2016) and by SORA in a classical EU model where the utility function is twice differentiable and concave at all wealth levels. This is considered in the following proposition.

Proposition 2. The optimal proportion of insurance coverage $\alpha_{r}^{*}$ that maximizes Equation (11) is always larger than the optimal proportion of insurance coverage $\alpha^{*}$ implied by a conventional utility function $u$ that exhibits SORA at all wealth levels.

Proof. Consider a conventional utility function of terminal wealth $u(w)$ that is always twice differentiable, with $u^{\prime}(w)>0$ and $u^{\prime \prime}(w)<0$ as implied by SORA. The optimal proportion $\alpha^{*} \in[0,1]$ of insurance coverage induced by $u(w)$ is found by maximization of $U(\alpha)$ defined in Equation (14). Owing to the concavity of $u, \alpha^{*}$ is the unique solution to

$$
(1+\eta) U^{\prime}\left(\alpha^{*}\right)=0
$$

where $\eta>0$ is the relative weight of gain-loss utility that appears in Equation (2). 
For a DM with reference-dependent utility expressed by Equation (1), the optimal proportion of insurance coverage is determined by the sign of $V^{\prime}(\alpha)$ in Equation (13). Evaluating the latter at $\alpha^{*}$ and subtracting the LHS of Equation (19) gives

$$
V^{\prime}\left(\alpha^{*}\right)-(1+\eta) U^{\prime}\left(\alpha^{*}\right)=\eta(\lambda-1) \int_{l^{r}}^{\bar{l}} u^{\prime}\left(w\left(l, \alpha^{*}\right)\right)\left[l-(1+k) \mu_{L}\right] d F_{L}
$$

which is positive under loss aversion because $\lambda>1$. It follows that the optimal proportion $\alpha_{r}^{*}$ of insurance coverage in the reference-dependent utility model expressed by Equation (1) is larger than $\alpha^{*}$.

Intuition would suggest that an increase in either loss aversion or in the gain-loss component of the utility function stimulates a higher demand for insurance coverage. The next proposition shows that this indeed the case.

Proposition 3. For a DM with reference dependent utility expressed by Equation (11) and target expressed by Equation (4), the optimal proportion of insurance coverage $\alpha_{r}^{*}$ increases as either $\lambda$ or $\eta$ increases.

Proof. Evaluating the first-order condition expressed by Equation (13) at the optimal coverage $\alpha_{r}^{*}$ gives

$$
V^{\prime}\left(\alpha_{r}^{*}\right)=(1+\eta) U^{\prime}\left(\alpha_{r}^{*}\right)+(\lambda-1) \eta \int_{l^{r}}^{\bar{l}} u^{\prime}\left(w\left(l, \alpha_{r}^{*}\right)\right)\left(l-l^{r}\right) d F_{L}(l)=0
$$

Differentiating $V^{\prime}\left(\alpha_{r}^{*}\right)$ in $\lambda$ gives

$$
\frac{\partial V^{\prime}}{\partial \lambda}=\eta \int_{l^{r}}^{\bar{l}} u^{\prime}\left(w\left(l, \alpha_{r}^{*}\right)\right)\left(l-l^{r}\right) d F_{L}>0
$$

which, in view of the concavity of $V(\alpha)$, implies that $\alpha_{r}^{*}$ is increasing in $\lambda$.

Differentiating $V^{\prime}\left(\alpha_{r}^{*}\right)$ in $\eta$ gives

$$
\frac{\partial V^{\prime}}{\partial \eta}=U^{\prime}\left(\alpha_{r}^{*}\right)+(\lambda-1) \int_{l^{r}}^{\bar{l}} u^{\prime}\left(w\left(l, \alpha_{r}^{*}\right)\right)\left(l-l^{r}\right) d F_{L} .
$$

The condition expressed by Equation (21) implies

$$
U^{\prime}\left(\alpha_{r}^{*}\right)=-(\lambda-1) \frac{\eta}{1+\eta} \int_{l^{r}}^{\bar{l}} u^{\prime}\left(w\left(l, \alpha_{r}^{*}\right)\right)\left(l-l^{r}\right) d F_{L}(l)
$$

Plugging this into Equation (22) yields:

$$
\frac{\partial V^{\prime}}{\partial \eta}=(\lambda-1) \frac{\eta^{2}}{1+\eta} \int_{l^{r}}^{\bar{l}} u^{\prime}\left(w\left(l, \alpha_{r}^{*}\right)\right)\left(l-l^{r}\right) d F_{L}(l)>0
$$

which, in view of the concavity of $V(\alpha)$, implies that $\alpha_{r}^{*}$ is increasing in $\eta$.

As shown in Section 2, increasing either $\lambda$ or $\eta$ entails an increase in the first-order term of the total risk premium at the reference point $r$.

The result of the above proposition is consistent with a well-established stream of the portfolio selection literature in which reference dependence and loss aversion are shown to determine the increasing demand for capital protection (Bertrand and Prigent (2011), for instance, make this point for constant proportion portfolio insurance (CPPI) strategies that offer increased protection against downside risk exposure). However, contrary to the mainstream thinking in the field, we here consider a reference-dependent utility function expressed by Equation (3) that has no convex portions. Our results 
may consequently be interpreted as direct implications of FORA as they do not presume risk loving attitudes below the reference point $r$.

As previously mentioned, the choice of $r$ is of crucial importance to reference-dependent utility models. For this reason, we here investigate the behavior of the optimal insurance coverage $\alpha_{r}^{*}$ as a function of the target $r$ taken into account.

Based on the condition expressed by Equation (27), the final wealth of a DM who insures a fraction $\alpha$ of the potential loss $L$ is given by $W(L, \alpha)=w_{0}-L+\alpha L-(1+k) \alpha \mu_{L}$. Hence, for a general target $r$, it follows that

$$
W \geq r \quad \Leftrightarrow \quad L \leq \frac{w_{0}-r-(1+k) \alpha \mu_{L}}{1-\alpha} \triangleq l_{r, k}
$$

Consequently,

$$
r \lesseqgtr w_{0}-(1+k) \mu_{L} \quad \Leftrightarrow \quad l_{r, k} \gtreqless(1+k) \mu_{L} .
$$

Let $\widetilde{r} \triangleq w_{0}-(1+k) \mu_{L}$ and, as previously, $l^{r} \triangleq(1+k) \mu_{L}$.

A loss-averse DM chooses the optimal proportion of insurance coverage $\alpha_{r}^{*} \in[0,1]$ by maximizing the expected utility of his/her final wealth:

$$
\begin{aligned}
V(\alpha) & =E[v(W, r)]=E\left\{u(W)+\Re^{\eta, \lambda}[u(W)-u(r)]\right\} \\
& =\int_{0}^{l_{r, k}}\{u(w(l, \alpha))+\eta[u(w(l, \alpha))-u(r)]\} d F_{L}(l) \\
& +\int_{l_{r, k}}^{\bar{l}}\{u(w(l, \alpha))+\lambda \eta[u(w(l, \alpha))-u(r)]\} d F_{L}(l) .
\end{aligned}
$$

Proposition 4. The optimal proportion $\alpha_{r}^{*}$ of insurance coverage that maximizes Equation (23) is (non-strictly) decreasing in $r$ for $r \leq \widetilde{r}$ and (non-strictly) increasing in $r$ for $r \geq \widetilde{r}$.

Proof. Differentiating Equation (23) with respect to $\alpha$ and following the same steps as those outlined in previous developments, we obtain

$$
V^{\prime}(\alpha)=(\eta+1) U^{\prime}(\alpha)+(\lambda-1) \eta \int_{l_{r, k}}^{\bar{l}} u^{\prime}(w)\left(l-(1+k) \mu_{L}\right) d F_{L}(l),
$$

where the first term is independent of the choice of $r$. It follows that

$$
\frac{\partial V^{\prime}}{\partial r}=\frac{(\lambda-1) \eta}{1-\alpha} u^{\prime}(r)\left(l_{r, k}-(1+k) \mu_{L}\right) f_{L}\left(l_{r, k}\right) \quad\left\{\begin{array}{cl}
<0 ; & r>\widetilde{r} \\
=0 ; & r=\widetilde{r} \\
>0 ; & r<\widetilde{r}
\end{array}\right.
$$

so $V^{\prime}$ is decreasing in $r$ for $r>\widetilde{r}$ and increasing in $r$ for $r<\widetilde{r}$. The argument above together with the concavity of $V(\alpha)$ in $\alpha$ implies the result.

As shown by the above result, our choice of reference point $\widetilde{r}$ identifies a crucial level of wealth for loss-averse decision-makers. In particular, compared to the frequently used "status quo" $\left(r=w_{0}\right)$, the optimal insurance proportion related to the safe alternative $\widetilde{r}$ is lower or equal.

\section{Deductible Insurance}

We now suppose that insurance is available in the form of a straight deductible policy. This repays the $\mathrm{DM}$ for losses in excess of a predefined deductible level $D \geq 0$, according to the indemnity function:

$$
I(L)=\max \{0, L-D\} .
$$


The price for obtaining deductible $D \geq 0$ is specified by the insurance premium, which is assumed proportional to the expected indemnity:

$$
\pi_{D}=(1+k) E[I(L)]=(1+k) \int_{D}^{\bar{l}}(l-D) d F_{L}(l)
$$

where $k>0$ is the loading factor, and

$$
\frac{\partial \pi_{D}}{\partial D}=-(1+k)\left[1-F_{L}(D)\right]
$$

As argued in Schlesinger (1985), at the agent's choice of a deductible level $D$, it is reasonable to assume

$$
\frac{\partial \pi_{D}}{\partial D}>-1
$$

which implies that the extra cost of lowering the deductible by 1 unit does not exceed the increase in final wealth when the realized loss $l$ exceeds $D$ (see Footnote 1 in Schlesinger (1985)).

The DM now faces a kinked payoff function:

$$
w(l, D)=\left\{\begin{array}{lll}
w^{+}=w_{0}-\pi_{D}-l & \text { if } l \leq D \\
w^{-}=w_{0}-\pi_{D}-D & \text { if } l>D
\end{array}\right.
$$

with

$$
\begin{array}{ll}
\frac{\partial w^{+}}{\partial D}=(1+k)\left[1-F_{L}(D)\right] & >0 \\
\frac{\partial w^{-}}{\partial D}=(1+k)\left[1-F_{L}(D)\right]-1 & <0
\end{array}
$$

where the last inequality holds at the agent's choice of a deductible $D$ as a consequence of the condition expressed by Equation (26).

Based on the reference-dependent utility expressed by Equation (1), the DM chooses the optimal deductible $D^{*}$ by maximizing the expected utility of his/her final payoff:

$$
\begin{aligned}
H(D) & =E[v(W(L ; D))] \\
& =\int_{0}^{l^{r}} v\left(w^{+}\right) d F_{L}+\int_{l^{r}}^{D} v\left(w^{+}\right) d F_{L}+\int_{D}^{\bar{l}} v\left(w^{-}\right) d F_{L} \\
& =\int_{0}^{l^{r}}\left[(1+\eta) u\left(w^{+}\right)-\eta u(r)\right] d F_{L}+\int_{l^{r}}^{D}\left[(1+\lambda \eta) u\left(w^{+}\right)-\lambda \eta u(r)\right] d F_{L} \\
& +\left[1-F_{L}(D)\right]\left[(1+\lambda \eta) u\left(w^{-}\right)-\lambda \eta u(r)\right]
\end{aligned}
$$

where $r=w_{0}-(1+k) \mu_{L}$ is the agent's reference wealth, as specified in Equation (4), and $l^{r}=w_{0}-\pi_{D}-r$ is the corresponding level of loss, with $0 \leq l^{r} \leq D$.

Assuming that $F_{L}$ is continuous at $l^{r}$ and $D$, and denoting the corresponding density function by $f_{L}$, we have

$$
\begin{aligned}
H^{\prime}(D) & =\left[1-F_{L}(D)\right]\left\{( 1 + k ) \left[(1+\eta) \int_{0}^{l^{r}} u^{\prime}\left(w^{+}\right) d F_{L}\right.\right. \\
& \left.\left.+(1+\lambda \eta) \int_{l^{r}}^{D} u^{\prime}\left(w^{+}\right) d F_{L}+(1+\lambda \eta)\left(1-F_{L}(D)\right) u^{\prime}\left(w^{-}\right)\right]-(1+\lambda \eta) u^{\prime}\left(w^{-}\right)\right\}
\end{aligned}
$$


for any $D>0$. After some calculation and rearrangement, this gives the following first-order condition for EU maximization at an interior point:

$$
H^{\prime}(D)=\left[1-F_{L}(D)\right]\left\{(1+k) E\left[v^{\prime}(W(L, D))\right]-(1+\lambda \eta) u^{\prime}\left(w^{-}\right)\right\}=0 .
$$

Using Equation (25), Equation (31) can be written as

$$
-\frac{\partial \pi_{D}}{\partial D} \int_{0}^{D} u^{\prime}\left(w^{+}\right) d F_{L}+\underbrace{\frac{\partial \pi_{D}}{\partial D} \frac{\eta(\lambda-1)}{1+\lambda \eta} \int_{0}^{l^{r}} u^{\prime}\left(w^{+}\right) d F_{L}}_{<0}+\left(-\frac{\partial \pi_{D}}{\partial D}-1\right)\left[1-F_{L}(D)\right] u^{\prime}\left(w^{-}\right)=0 .
$$

According to Schlesinger (2013), the first and third term in the above equation can be interpreted, for an agent with utility function $u$, as the marginal benefit of premium savings from increasing $D$ conditional on a loss lower than $D$, and the marginal cost of a higher deductible given that the loss exceeds $D$, respectively. The second addend in Equation (32) is an additional term due to the gain-loss component of Guo's model that lowers the marginal benefit when the loss is lower than $l^{r}$, taking into account the behavioral parameters $\lambda, \eta$. This extra term depends on the ratio $\frac{\eta(\lambda-1)}{1+\lambda \eta}$ that is increasing in each parameter $\eta, \lambda$ and tends to zero when either $\eta \rightarrow 0^{+}$or $\lambda \rightarrow 1^{+}$, converging to the case of a SORA agent with conventional utility $u$ (see Equation (16) in Schlesinger (2013)).

Evaluating $H^{\prime \prime}(D)$ at an interior solution $D_{r}^{*} \in(0, \bar{l})$ of Equation (31) gives

$$
\begin{aligned}
H^{\prime \prime}\left(D_{r}^{*}\right) & =\left[1-F_{L}\left(D_{r}^{*}\right)\right]\left\{( 1 + k ) \left[(1+\eta) \int_{0}^{l^{r}} u^{\prime \prime}\left(w^{+}\right) \frac{\partial w^{+}}{\partial D} d F_{L}+\right.\right. \\
& +(1+\lambda \eta) \int_{l^{r}}^{D_{r}^{*}} u^{\prime \prime}\left(w^{+}\right) \frac{\partial w^{+}}{\partial D} d F_{L}-\eta(\lambda-1) \frac{\partial l^{r}}{\partial D} u^{\prime}(r) f_{L}\left(l^{r}\right) \\
& \left.\left.+(1+\lambda \eta) u^{\prime \prime}\left(w^{-}\right) \frac{\partial w^{-}}{\partial D}\left(1-F_{L}\left(D_{r}^{*}\right)\right)\right]-(1+\lambda \eta) u^{\prime \prime}\left(w^{-}\right) \frac{\partial w^{-}}{\partial D}\right\}
\end{aligned}
$$

where $\frac{\partial l^{r}}{\partial D}=(1+k)\left[1-F_{L}(D)\right]>0$. Based on Equation (28) and on the concavity of $u$, the above expression is negative if

$$
(1+k)\left[1-F_{L}\left(D_{r}^{*}\right)\right]-1<0
$$

at all values $D_{r}^{*} \in(0, \bar{l})$ that solve the first-order condition expressed by Equation (31). The inequality in Equation (33) holds at the agent's choice of an optimal deductible as a consequence of Equation (26). It follows that any $D_{r}^{*} \in(0, \bar{l})$ that solves the first-order condition expressed by Equation (31) identifies an optimal insurance policy (as guaranteed by Equation (33)).

Proposition 5. Consider a FORA agent with reference-dependent utility expressed by Equation (1) and reference wealth $r=w_{0}-(1+k) \mu_{L}$. The agent chooses the optimal level of deductible insurance, $D_{r}^{*}, b y$ maximizing the expected utility specified in Equation (29). Assume the loading factor $k$ satisfies

$$
0<k<\frac{q}{1-q}
$$

where $q=F_{L}(0)=P(L=0)>0$.

If

$$
\frac{1+\lambda \eta}{1+\eta}>\frac{(1+k) q}{1-(1+k)(1-q)}
$$

the agent will purchase full insurance coverage, i.e., $D_{r}^{*}=0$. If the inequality in Equation (35) is reversed, then $D_{r}^{*}>0$. 
Proof. In view of Schlesinger's condition expressed by Equation (26), it is reasonable to study an agent's decision to purchase full coverage when

$$
\lim _{D \rightarrow 0} \frac{\partial \pi_{D}}{\partial D}=\lim _{D \rightarrow 0}\left\{-(1+k)\left[1-F_{L}(D)\right]\right\}>-1,
$$

which implies that the agent could still derive an economic benefit when the level of deductible is lowered to zero. The condition expressed by Equation (36) is satisfied if $F_{L}$ has a point mass $q>0$ at 0 and the loading factor $k$ is bounded above as in Equation (34). In this case, taking the limit of Equation (30) for $D \rightarrow 0$ gives

$$
\lim _{D \rightarrow 0} H^{\prime}(D)=(1-q) u^{\prime}\left[w_{0}-(1+k) \mu_{L}\right]\{(1+\eta)(1+k) q-(1+\lambda \eta)[1-(1+k)(1-q)]\},
$$

which is negative under the condition expressed by Equation (35). Since Equation (36) implies Equation (33) in $(0, \bar{l})$, it follows that $D_{r}^{*}=0$ is a constrained optimum (when overinsurance is prohibited). If the inequality in the condition expressed by Equation (35) is reversed, the sign of Equation (37) is positive and $D_{r}^{*}>0$, i.e., partial coverage is preferable.

In the past decade, a number of empirical and experimental studies on insurance-purchasing decisions have extensively documented that some customers purchase low deductibles despite costs significantly above fair price. However, fitting these choices to a standard EU model and assuming that preferences exhibit SORA at all wealth levels yields unrealistic large measures of absolute risk aversion (Sydnor 2010). This is typically regarded as a major puzzle in insurance economics (Schmidt 2016).

The result of the above proposition shows that FORA is a plausible explanation of consumers' preferences for low deductibles, possibly leading to the purchase of full coverage even at a loaded price. As shown in the condition expressed by Equation (35), this decision depends on the loss aversion coefficient $(1+\lambda \eta) /(1+\eta)$ and on the relative probabilities of experiencing/not experiencing a loss. The role of these probabilities has already emerged in a study of insurance demand that Schmidt (2016) has recently carried out by graphical analyses in a different framework, based on a single-argument value function that is specified as a power function of gains and losses (as in conventional prospect theory). Interestingly, Schmidt (2016) also considers the "safe alternative" (i.e., the fully insured position) as a possible reference point, but restricts attention to binary risks and fair insurance contracts. Differently than Schmidt (2016), we model reference dependence using a two-argument utility function expressed by Equation (3) that has no convex portions, and we derive results for a general loss distribution with a positively loaded insurance premium. Our analysis can thus help explain why, in real-world circumstances, risk-averse agents may be willing to purchase full coverage even at costs that exceed the expected value of the insurance contract.

As shown in Equation (32), the presence of FORA in Guo's model alters the economic trade-off of a conventional SORA agent by lowering the marginal benefit of increasing the deductible level, given that the loss is lower than $D$. The following proposition shows that FORA stimulates insurance demand by inducing the choice of a lower deductible relative to SORA.

Proposition 6. Denote the optimal deductible for a FORA agent by $D_{r}^{*}$ with reference-dependent utility expressed by Equation (3) and reference wealth $r=w_{0}-(1+k) \mu_{L}$. If the condition expressed by Equation (33) is verified and $H$ is concave in $D$, then $D_{r}^{*} \leq D^{*}$, where $D^{*}$ is the optimal deductible implied by a conventional utility function $u(w)$ that exhibits SORA at all wealth levels. 
Proof. If we consider a twice differentiable utility function $u$ with $u^{\prime}>0$ and $u^{\prime \prime}<0$, the optimal deductible is found by maximization of the expected utility

$$
U(D)=E[u(W(L, D))]=\int_{0}^{D} u\left(w^{+}\right) d F_{L}+\left[1-F_{L}(D)\right] u\left(w^{-}\right)
$$

where $w^{+}, w^{-}$are defined by Equation (27). Since we assume proportional insurance pricing, the first-order condition

$$
U^{\prime}(D)=\left[1-F_{L}(D)\right]\left\{(1+k) E\left[u^{\prime}(W(L, D))\right]-u^{\prime}\left(w^{-}\right)\right\}=0
$$

identifies a unique global maximizer $D^{*}$, which is the optimal deductible for a SORA agent (cf. Meyer and Ormiston (1999)). It follows that

$$
(1+\eta) U^{\prime}\left(D^{*}\right)=0
$$

For a FORA agent with the reference-dependent utility specified in Equation (1), the optimal deductible is determined by the sign of $H^{\prime}(D)$ in Equation (30) under the condition expressed by Equation (33). Evaluating $H^{\prime}$ at $D^{*}$ and subtracting the LHS of Equation (38) gives, after some manipulation,

$$
\begin{aligned}
H^{\prime}\left(D^{*}\right) & -(1+\eta) U^{\prime}\left(D^{*}\right) \\
& =\eta(\lambda-1)\left\{\frac{\partial w^{+}}{\partial D} \int_{l^{r}}^{D^{*}} u^{\prime}\left(w^{+}\right) d F_{L}+\frac{\partial w^{-}}{\partial D}\left[1-F_{L}\left(D^{*}\right)\right] u^{\prime}\left(w^{-}\right)\right\} \\
& =\eta(\lambda-1)\left[1-F_{L}\left(D^{*}\right)\right]\left\{(1+k)\left[\int_{l^{r}}^{D^{*}} u^{\prime}\left(w^{+}\right) d F_{L}+\left(1-F_{L}\left(D^{*}\right)\right) u^{\prime}\left(w^{-}\right)\right]-u^{\prime}\left(w^{-}\right)\right\} .
\end{aligned}
$$

Since $u^{\prime}\left(w^{+}\right) \leq u^{\prime}\left(w^{-}\right)$for all $l \in\left[l^{r}, D^{*}\right]$, we have

$$
H^{\prime}\left(D^{*}\right)-(1+\eta) U^{\prime}\left(D^{*}\right) \leq \eta(\lambda-1)\left[1-F_{L}\left(D^{*}\right)\right] u^{\prime}\left(w^{-}\right)\left\{(1+k)\left[1-F_{L}\left(l^{r}\right)\right]-1\right\},
$$

which is negative if

$$
(1+k)\left[1-F_{L}\left(l^{r}\right)\right]-1<0 .
$$

Since $r=w_{0}-(1+k) \mu_{L}$ implies that $0 \leq l^{r} \leq D_{r}^{*}$, the condition expressed by Equation (33) implies Equation (39). It follows that the optimal deductible $D_{r}^{*}$ under FORA is lower than the optimal deductible $D^{*}$ under SORA, i.e., FORA stimulates the demand for insurance coverage.

\section{Deductible or Proportional?}

A cornerstone result in the theory of insurance economics is Arrow's theorem of the deductible. The theorem states that a straight deductible policy is the optimal insurance contract for all risk-averse DMs whenever the insurer's costs are proportional to the indemnity payment and the insurer is risk-neutral (Arrow 1974).

In this section, we extend Arrow's fundamental result to FORA preferences, building on a stochastic dominance rule for comparing risks in the presence of a reference point that has been recently proposed by Guo et al. (2016).

Definition 1. (Guo et al. 2016) Consider the reference-dependent utility specified in Equation (1) and assume $u \in \mathcal{U}^{2}$, where

$$
\mathcal{U}^{2}=\left\{u:(-\infty, \infty) \rightarrow \Re \mid u^{\prime} \geq 0, u^{\prime \prime}<0\right\}
$$


Assume $\eta^{*} \geq 0, \lambda^{*} \geq 1$ are pre-specified lower bounds for the parameters $\eta, \lambda$ and the reference point $r$ is given. A random variable $W_{1}$ is said to second-order-stochastically dominate another random variable $W_{2}$ relative to $r$, written as $W_{1} S S D^{r} W_{2}$, if

$$
E\left[v\left(W_{1} ; r, u\right)\right] \geq E\left[v\left(W_{2} ; r, u\right)\right]
$$

for all $u \in \mathcal{U}^{2}, \eta \geq \eta^{*}, \lambda \geq \lambda^{*}$.

As proved in Guo et al. (2016), conventional second-order stochastic dominance (SSD) is a sufficient condition for $S S D^{r}$ relative to a fixed reference point, as illustrated in the following proposition.

Proposition 7. (Guo et al. 2016) Let the reference point $r$ be fixed. For any two random variables $W_{1}$ and $W_{2}$, $W_{1} S S D W_{2}$ implies $W_{1} S S D^{r} W_{2}$, but the converse is not true.

Based on this result, we now extend the validity of Arrow's theorem to the reference-dependent utility model expressed by Equation (1).

Proposition 8. Consider a FORA agent with reference-dependent utility expressed by Equation (1) and a fixed reference wealth $r=w_{0}-(1+k) \mu_{L}$, as specified in Equation (4). As a hedge against a potential random loss $L$ valued in $[0, \bar{l}]$, the agent selects an insurance contract that stipulates an indemnity $I(L)$ and requires the payment of a premium $\pi=(1+k) E[I(L)]$, with $k>0$. If $I(l)$ is non-decreasing and $0 \leq I(l) \leq l$ for all $l$, the optimal insurance contract contains a straight deductible $D$, i.e., $I(L)=\max (L-D, 0)$.

Proof. It is well known (see, e.g., Eeckhoudt and Gollier (1992); Gollier and Schlesinger (1996)) that, for a given insurance premium $\pi=(1+k) E[I(L)]$, the indemnity schedule $I(L)=\max (L-D, 0)$ second-order-stochastically dominates any non-negative and non-decreasing indemnity function $\tilde{I}(L)$ with the same premium $\pi$. By Proposition 7 above, $I(L) S S D \tilde{I}(L)$ implies that $I(L) S S D^{r} \tilde{I}(L)$. Therefore, according to Definition 1, the deductible contract is preferred by all FORA agents with reference-dependent utility expressed by Equation (1) and a fixed target $r$ specified in Equation (4). Since we can make this argument for any level $\pi$ of the insurance premium, this concludes the proof.

The logic behind deductible insurance is that of concentrating coverage on the states with the largest losses. Originally, Arrow proved that this logic is optimal for all risk-averse EU-maximizers. Other authors (see Gollier and Schlesinger (1996), for a detailed overview) have subsequently extended Arrow's result beyond the classic EU framework using preference functionals consistent with firstand second-degree stochastic dominance.

In the above proposition, we prove that the validity of Arrow's theorem is preserved under FORA in view of the coherence between the reference-dependent utility function expressed by Equation (3) and the definition of second-degree stochastic dominance with respect to a fixed reference point provided in Guo et al. (2016). The question of whether this result could be extended to other types of reference-dependent preferences (with possibly stochastic reference points) is still an open one, and we leave it for further research.

\section{Discussion and Conclusions}

Some decision-makers pay attention not only to their final wealth but also to its position with respect to a reference value.

Recently, Guo et al. (2016) have suggested an interesting model of choice belonging to that line of research and they have looked at its implications for the stochastic dominance literature. An interesting feature of the model above is that it introduces loss aversion and gain loving without affecting the 
local index of risk aversion away from the reference point. In this way, one can better isolate the pure effect of these attitudes upon optimal choices under risk.

In the present paper, we have examined how insurance demand would behave in such an environment. It appears that the reference level $r$ adopted by the decision-maker plays a major role. As recently argued in Schmidt (2016), a possible choice of the reference level is the so-called safe alternative, i.e., the sure amount of wealth, the decision-maker could obtain if $\mathrm{s} / \mathrm{he}$ were to select full insurance. For values of $r$ equal to the safe alternative, we have found that, under a strictly positive loading, the second component of the utility function besides final wealth always stimulates insurance demand.

The proposed model yields plausible explanations for a range of consumer choices observed in real insurance markets. In particular, decision-makers within the reference-dependent utility model of Guo et al. (2016) will (a) always choose a lower deductible (or a higher coinsurance rate) relative to classic risk-averse EU-maximizers and (b) be willing to purchase full insurance even at a loaded price, subject to appropriate conditions on their degree of loss aversion. Interestingly, in the case of proportional insurance, these conditions are related to the Omega measure, a performance index that has been widely used in mathematical finance and in the portfolio management literature (see, e.g., Bertrand and Prigent (2011) and the references therein).

Whereas the mainstream of literature has focused on binary risks and/or fair insurance contracts (see, e.g., Schmidt (2016), for a recent contribution and an accurate literature review), in the present work we have considered a general loss distribution and a positively loaded insurance premium. Our results are consequently relevant for understanding the characteristics of real insurance markets, and provide a new perspective on consumers' tendency to over-insure modest risks (Sydnor 2010).

These findings add to a range of possible explanations that have been suggested in the literature to rationalize a surprisingly high demand for insurance, such as probability weighting (Jaspersen et al. 2018), consumption commitments (Chetty 2006), and menu effects (Kamenica 2008).

It is worth mentioning that Guo's model presumes separability between consumption utility and gain-loss utility, which adds tractability to the problem. Alternative points of view have been recently proposed in the literature. In particular, Gollier (2016) integrates consumption utility and regret within a non-separable model, whereas Andersen et al. (2018) argue in favor of more flexible models that allow for partial rather full asset integration.

Another specificity of Guo's model is the assumption that initial wealth is fixed and known to the agent. This assumption has been discussed and relaxed, for instance, by Barberis et al. (2006), who have emphasized the role of background risks from extra-experimental income or wealth. However, when these background risks are independent of the insurable risk, they induce diversification benefits that may offset first-order risk aversion. A promising direction to develop a model with multiple sources of risks may be found, for instance, in Dionne and Li (2014), who suggest that foreground and background risks may not be independent but rather associated in a form of positive/negative expectation dependence.

Author Contributions: The authors contributed equally to this work.

Funding: This research was supported by the Chair: Individuals and Risk: analysis and market response (Les particuliers face au risque: analyse et réponse des marchés), as well as the Chair's partners: Fondation du Risque, Université Paris-Dauphine, ENSAE and Groupama (year 2015).

Acknowledgments: We thank two referees for their valuable comments and suggestions that contributed to improve the paper.

Conflicts of Interest: The authors declare no conflict of interest.

\section{References}

Abdellaoui, Mohammed, Han Bleichrodt, and Corina Paraschiv. 2007. Loss Aversion under Prospect Theory: A Parameter-Free Measurement. Management Science 53: 1659-74. [CrossRef] 
Andersen, Steffen, James C. Cox, Glenn W. Harrison, Morten Lau, E. Elisabet Rutström, and Vjollca Sadiraj. 2018. Asset integration, and attitudes to risk: Theory and evidence. Review of Economics and Statistics, accepted for publication._a_00719. [CrossRef]

Arrow, Kenneth J. 1974. Essays in the Theory of Risk Bearing. Amsterdam: North-Holland.

Baillon, Aurélien, Han Bleichrodt, and Vitalie Spinu. 2016. Searching for the Reference Point. Working Paper. Rotterdam: Erasmus School of Economics, Erasmus University Rotterdam.

Barberis, Nicholas, Ming Huang, and Richard H. Thaler. 2006. Individual preferences, monetary gambles, and stock market participation: A case for narrow framing. American Economic Review 96: 1069-90. [CrossRef]

Bernard, Carole, and Mario Ghossoub. 2010. Static portfolio choice under cumulative prospect theory. Mathematics and Financial Economics 2: 277-310. [CrossRef]

Bertrand, Philippe, and Jean-luc Prigent. 2011. Omega performance measure and portfolio insurance. Journal of Banking and Finance 35: 1811-23. [CrossRef]

Borch, Karl Henrik. 1960. The safety loading of reinsurance premiums. Scandinavian Actuarial Journal 1960: 163-84. [CrossRef]

Borch, Karl Henrik. 1962. Equilibrium in a Reinsurance Market. Econometrica 30: 424-44. [CrossRef]

Borch, Karl Henrik. 1990. Economics of Insurance. Amsterdam: North-Holland.

Chetty, Raj. 2006. A new method of estimating risk aversion. American Economic Review 96: 1821-34. [CrossRef]

Cox, James C., and Vjollca Sadiraj. 2006. Small-and large-stakes risk aversion: Implications of concavity calibration for decision theory. Games and Economic Behavior 56: 45-60. [CrossRef]

Cox, James C., and Vjollca Sadiraj. 2008. Risky Decisions in the Large and in the Small: Theory and Experiment. In Risk Aversion in Experiments. Edited by James C. Cox and Glenn W. Harrison. Bingley: Emerald Group Publishing Limited, pp. 9-40.

Davies, Greg B., and Stephen E. Satchell. 2007. The behavioural components of risk aversion. Journal of Mathematical Psychology 51: 1-13. [CrossRef]

Denneberg, Dieter. 1990. Distorted probabilities and insurance premiums. Methods of Operations Research 63: 3-5.

Dionne, Georges, and Jingyuan Li. 2014. When can expected utility handle first-order risk aversion? Journal of Economic Theory 154: 403-22. [CrossRef]

Doherty, Neil A., and Louis Eeckhoudt. 1995. Optimal insurance without expected utility: The dual theory and the linearity of insurance contracts. Journal of Risk and Uncertainty 10: 157-79. [CrossRef]

Eeckhoudt, Louis, and Christian Gollier. 1992. Les Risques Financiers: Evaluation, Gestion, Partage. Paris: Ediscience International.

Eeckhoudt, Louis, Anna Maria Fiori, and Emanuela Rosazza Gianin. 2016. Loss Averse Preferences and Portfolio Choices: An Extension. European Journal of Operational Research 249: 224-30. [CrossRef]

Gollier, Christian. 2016. Aversion to Risk of Regret and Preference for Positively Skewed Risks (No. 16-646). Toulouse: Toulouse School of Economics.

Gollier, Christian, and Harris Schlesinger. 1996. Arrow's theorem on the optimality of deductibles: A stochastic dominance approach. Economic Theory 7: 359-63. [CrossRef]

Gul, Faruk. 1991. A theory of disappointment aversion. Econometrica: Journal of the Econometric Society 59: 667-86. [CrossRef]

Guo, Dongmei, Yi Hu, Shouyang Wang, and Lin Zhao. 2016. Comparing Risks with Reference Points: A Stochastic Dominance Approach. Insurance: Mathematics and Economics 70: 105-16. [CrossRef]

Harrison, Glenn W., and J. Todd Swarthout. 2016. Cumulative Prospect Theory in the Laboratory: A Reconsideration. Working Paper, 2. Atlanta: Center for the Economic Analysis of Risk, Georgia State University.

Jarrow, Robert, and Feng Zhao. 2006. Downside loss aversion and portfolio management. Management Science 52: 558-66. [CrossRef]

Jaspersen, Johannes G., Richard Peter, and Marc A. Ragin. 2018. The Role of Probability Weighting in Optimal Insurance Demand: A Double-Edged Sword. Working Paper. Available online: https://www.uibk.ac.at/ credence-goods/events (accessed on 16 May 2018).

Kamenica, Emir. 2008. Contextual inference in markets: On the informational content of product lines. American Economic Review 98: 2127-49. [CrossRef]

Keating, Con, and William Shadwick. 2002. A universal performance measure. Journal of Performance Measurement 6: 59-84. 
Köbberling, Veronika, and Peter Wakker. 2005. An index of loss aversion. Journal of Economic Theory 122: 119-31. [CrossRef]

Köszegi, Botond, and Matthew Rabin. 2006. A model of reference-dependent preferences. The Quarterly Journal of Economics 121: 1133-65.

Köszegi, Botond, and Matthew Rabin. 2007. Reference-dependent risk attitudes. American Economic Review 97: 1047-73. [CrossRef]

Masatlioglu, Yusufcan, and Collin Raymond. 2016. A behavioral analysis of stochastic reference dependence. American Economic Review 106: 2760-82. [CrossRef]

Meyer, Jack, and Michael B. Ormiston. 1999. Analyzing the demand for deductible insurance. Journal of Risk and Uncertainty 18: 223-30. [CrossRef]

Mossin, Jan. 1968. Aspects of rational insurance purchasing. Journal of Political Economy 76: 533-68. [CrossRef]

Müller, Alfred, Marco Scarsini, Ilia Tsetlin, and Robert L. Winkler. 2016. Between first-and second-order stochastic dominance. Management Science 63: 2933-47. [CrossRef]

Murphy, Ryan O., and Robert H. ten Brincke. 2018. Hierarchical maximum likelihood parameter estimation for cumulative prospect theory: Improving the reliability of individual risk parameter estimates. Management Science 64: 308-26. [CrossRef]

Nilsson, Håkan, Jörg Rieskamp, and Eric-Jan Wagenmakers. 2011. Hierarchical Bayesian parameter estimation for cumulative prospect theory. Journal of Mathematical Psychology 55: 84-93. [CrossRef]

Peter, Richard, Andreas Richter, and Paul Thistle. 2017. Endogenous information, adverse selection, and prevention: Implications for genetic testing policy. Journal of Health Economics 55: 95-107. [CrossRef] [PubMed]

Schlesinger, Harris. 1985. Choosing a Deductible for Insurance Contracts: Best or Worst Insurance Policy? Journal of Risk and Insurance 52: 522-27. [CrossRef]

Schlesinger, Harris. 2013. The theory of insurance demand. In Handbook of Insurance, 2nd ed. Edited by Georges Dionne. New York: Springer, pp. 167-84.

Schmidt, Ulrich. 2016. Insurance demand under prospect theory: A graphical analysis. Journal of Risk and Insurance 83: 77-89. [CrossRef]

Schmidt, Ulrich, Chris Starmer, and Robert Sugden. 2008. Third-generation prospect theory. Journal of Risk and Uncertainty 36: 203-23. [CrossRef]

Schmidt, Ulrich, and Horst Zank. 2008. Risk aversion in cumulative prospect theory. Management Science 54: 208-16. [CrossRef]

Segal, Uzi, and Avia Spivak. 1990. First order versus second order risk aversion. Journal of Economic Theory 51: 111-25. [CrossRef]

Sugden, Robert. 2003. Reference-dependent subjective expected utility. Journal of Economic Theory 111: $172-91$. [CrossRef]

Sydnor, Justin. 2010. (Over) insuring modest risks. American Economic Journal: Applied Economics 2: 177-99. [CrossRef]

Wang, Shaun S., and Virginia R. Young. 1998. Ordering risks: Expected utility theory versus Yaari's dual theory of risk. Insurance: Mathematics and Economics 22: 145-61. [CrossRef]

Yaari, Menahem E. 1987. The Dual Theory of Choice Under Risk. Econometrica 55: 95-116. [CrossRef]

(c) 2018 by the authors. Licensee MDPI, Basel, Switzerland. This article is an open access article distributed under the terms and conditions of the Creative Commons Attribution (CC BY) license (http://creativecommons.org/licenses/by/4.0/). 